\title{
Wavefront Control for Extreme Adaptive Optics
}

\author{
L. A. Poyneer
}

This article was submitted to Astronomical Adaptive Optics Systems and Applications, San Diego, California, 08/03/2003 - 08/04/2003

\section{July 16, 2003}

Lawrence

Livermore

National

Laboratory

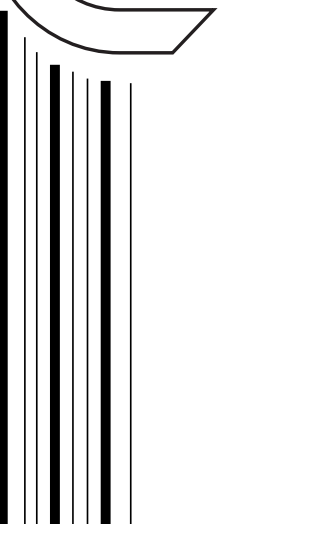


This document was prepared as an account of work sponsored by an agency of the United States Government. Neither the United States Government nor the University of California nor any of their employees, makes any warranty, express or implied, or assumes any legal liability or responsibility for the accuracy, completeness, or usefulness of any information, apparatus, product, or process disclosed, or represents that its use would not infringe privately owned rights. Reference herein to any specific commercial product, process, or service by trade name, trademark, manufacturer, or otherwise, does not necessarily constitute or imply its endorsement, recommendation, or favoring by the United States Government or the University of California. The views and opinions of authors expressed herein do not necessarily state or reflect those of the United States Government or the University of California, and shall not be used for advertising or product endorsement purposes. 


\title{
Wave-front control for extreme adaptive optics
}

\author{
Lisa A. Poyneer and Bruce Macintosh \\ Lawrence Livermore National Lab, Livermore, CA, USA
}

\begin{abstract}
Current plans for Extreme Adaptive Optics systems place challenging requirements on wave-front control. This paper focuses on control system dynamics, wave-front sensing and wave-front correction device characteristics. It may be necessary to run an ExAO system after a slower, low-order AO system. Running two independent systems can result in very good temporal performance, provided specific design constraints are followed. The spatiallyfiltered wave-front sensor, which prevents aliasing and improves PSF sensitivity, is summarized. Different models of continuous and segmented deformable mirrors are studied. In a noise-free case, a piston-tip-tilt segmented MEMS device can achieve nearly equivalent performance to a continuous-sheet DM in compensating for a static phase aberration with use of spatial filtering.
\end{abstract}

Keywords: adaptive optics, wave-front sensing, wave-front control

\section{INTRODUCTION}

Extreme Adaptive Optics (ExAO) requires new precision in wave-front control methods and pushes the technological limits of current hardware. The term ExAO encompasses systems that have large numbers of actuators and high control rates. This is necessary to achieve Strehl ratios near one and to enable high-contrast imaging. In this paper we will address three major issues for ExAO wave-front control. The first is due to technological limitations on current MEMS phase corrector stroke. Insufficient stroke may require that an ExAO system run after a low-order AO system which removes a substantial percentage of the wave-front phase aberration. We examine the case of two control loops running independently on the same phase aberration. This analysis shows that given adequate characterization of the low-order AO system, the ExAO system will successfully run afterwards. Modal removal or inter-system communication is not necessary.

A second concern for ExAO is how well the phase can be corrected given a specific deformable mirror (DM) or MEMS mirror. To address this question the influence function model for DM performance is assumed and a variety of continuous and segmented surface mirrors are analyzed in comparison to the ideal. Segmented MEMS introduce high-spatial-frequency content into the phase aberration. This content can be detrimental or even performance-limiting. Filtering the phase to mask this power (and prevent aliasing) makes the best segmented model perform almost as well in closed loop as the continuous DMs.

The prevention of aliasing is the third important ExAO topic discussed in this paper. Because the ExAO system will sample the phase during measurement, aliasing will occur in the general case. This can be prevented with use of a spatial filter before the wave-front sensor. We summarize the key results of the detailed Fourieroptics analysis of the spatially-filtered WFS (SFWFS). Results show that in closed-loop, noise-free AO operation on either reasonable atmospheric turbulence or segmented primary mirror phase errors, the use of the SFWFS reduces phase power by 50 to 100 times due to the prevention of aliasing.

\section{TEMPORAL CONTROL IN CLOSED LOOP}

The DM or MEMS mirror which is used to correct atmospheric turbulence must have adequate stroke (the physical distance the mirror surface can move.) A total stroke of 4 microns is necessary for correction of reasonable turbulence (with tip-tilt removed) on an 8 -meter aperture. ${ }^{1}$ This condition is met by the traditional DM technologies used in regular AO systems on large telescopes. However, currently available MEMS devices have only 2 microns stroke. ${ }^{2}$ It is possible that the 1000- to 4000-actuator class MEMS devices necessary for ExAO may not have sufficient stroke to correct atmospheric turbulence.

Send correspondence to Lisa A. Poyneer. E-mail: poyneer1@llnl.gov, Telephone: 19254233360 


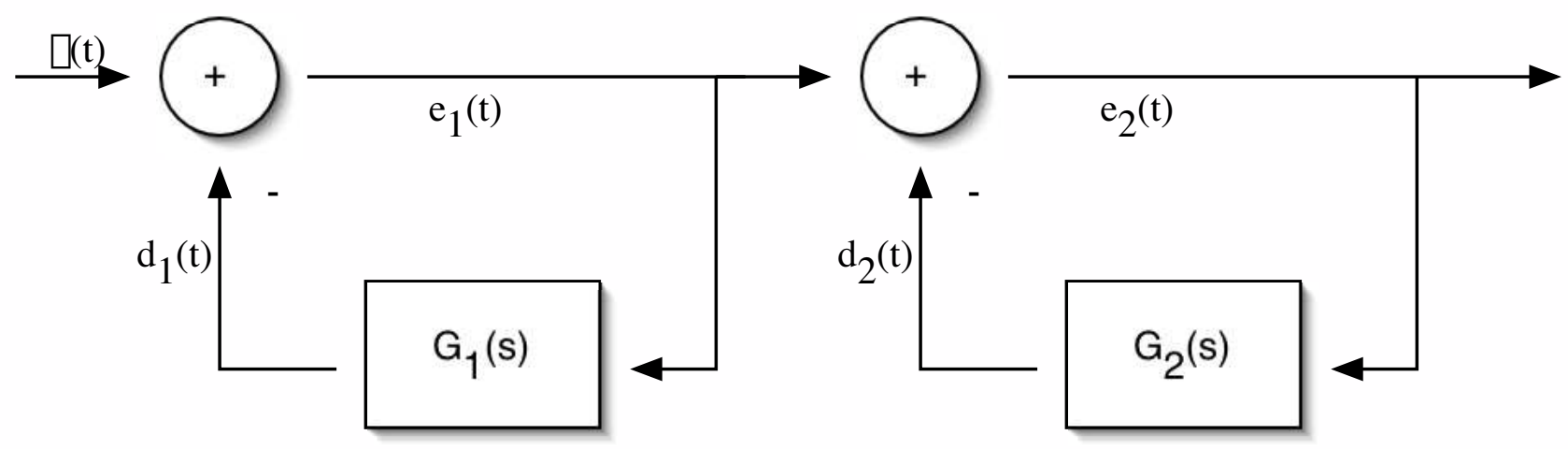

Figure 1. Block diagram for two AO control systems. The first AO system is noted by $G_{1}(s)$, and it produces error residual $e_{1}(t)$. The addition of ExAO system $G_{2}(s)$ after it is a simple cascade, producing error residual $e_{2}(t)$.

One option to get around the stroke limitation would be to use multiple DMs. Another solution is to run the ExAO system after a low-order AO system that is already installed on the telescope. This first AO system will remove a significant percentage of the low-spatial-frequency power, where the most stroke is necessary. This is analogous to the operation of separate tip-tilt and high-order correction loops with a tip-tilt mirror and a DM. However, in the ExAO case, it is reasonable to assume the the ExAO system will have no communication with the low-order AO running in front of it.

With two independent control systems correcting at the same time, care must be taken to ensure that no undesirable interactions or instabilities occur. This is less a concern for the ExAO system, which will 'see' everything that the low-order AO system is doing. The low-order system will be operating at a slower rate and will be unaware of any corrections to the phase downstream. The impact of running two AO systems at once needs to be carefully studied before system design. We have carried out such a study using a comprehensive discrete-time feedback control model for both systems. Below we present the fundamentals of the control system analysis and analyze how the two systems will perform as a whole.

\subsection{AO control system model}

For the purposes of this study we will consider the control of a single variable to illustrate temporal effects. We will not attempt to analyze the control of the entire phase in the pupil. For a detailed analysis of control system theory, see a standard text such as Control Systems Engineering. ${ }^{3}$ A basic feedback control system can be modeled as illustrated in Fig. 1. The input phase aberration $\phi(t)$, which changes with time, is corrected by the shape of the DM $d(t)$. The commands applied to the DM are determined in feedback through the system $G(s)$, which encompasses the entire control system. The system is driven not by the actual input $\phi(t)$ but by the error $e(t)$ that remains after correction by the DM. The system seeks to drive the error to zero. The error response of the system $E(j \omega)$ is the transfer from the input phase to the residual error. This is simply $1 /(1+G(j \omega))$. In our case this shows how much of the input phase aberration remains in the residual after correction with AO.

The AO system represented by $G(s)$ is actually quite complex. It is a discrete-time control system with a wave-front sensor and DM that 'sample and hold' to interface with continuous-time phase process. Work elsewhere has established the transfer functions for such a control system (see Madec ${ }^{4}$ for a clear summary), and we will simply state the final results. Using the Laplace and Z-transforms, the discrete and continuous are coupled and the Laplace transform of the $\mathrm{AO}$ system $G(s)$ is

$$
G(s)=\operatorname{Exp}[-\tau s]\left(\frac{1-\operatorname{Exp}[-s T]}{s T}\right)^{2} C(z=\operatorname{Exp}[s T])
$$

where $\tau$ is the compute delay, $T$ is the time of the sample and hold of the WFS and DM and $C(z)$ is the Z-transform of the discrete controller. At this point, because we wish to examine different types of discrete 

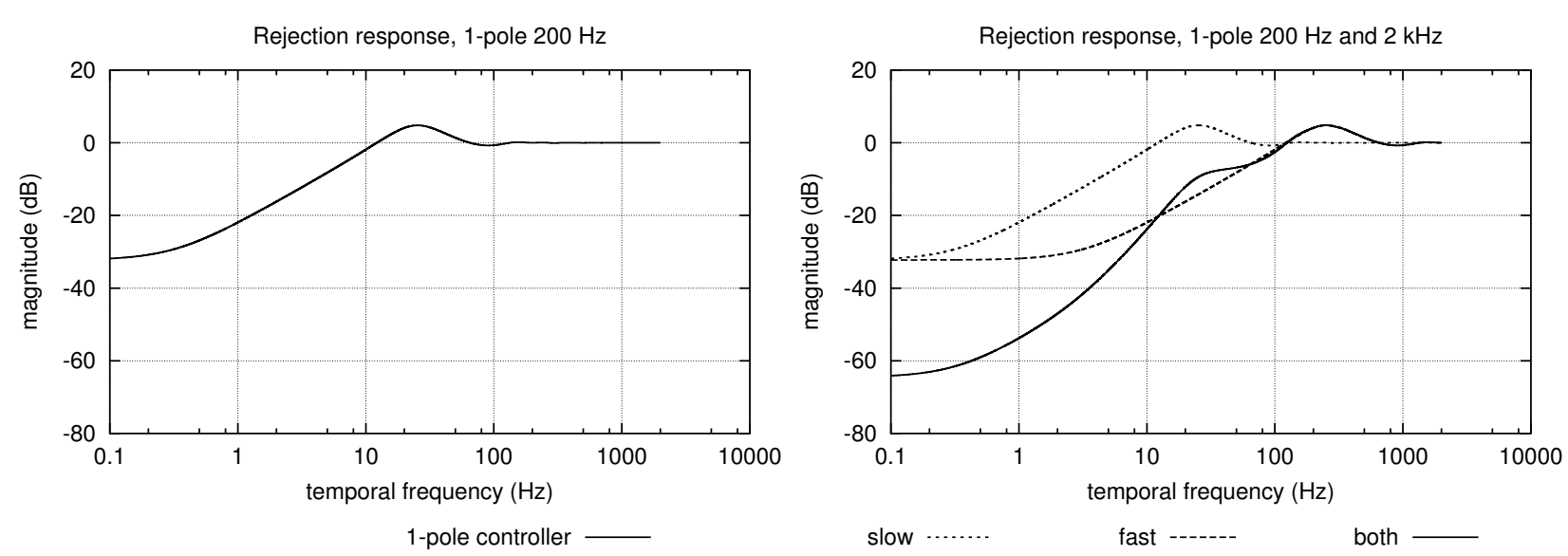

Figure 2. [Left panel]: Error response of a 1-pole AO system running at $200 \mathrm{~Hz}$. The correctible bandwidth is $12.2 \mathrm{~Hz}$. [Right panel]: The same slow system as other panel combined with a $2 \mathrm{kHz}$ ExAO system. The combined AO system error response is the sum of the two individual curves.

controllers, we will analyze the system as is. Because $G(s)$ is complex-valued, we can separate it into magnitude and phase. Evaluating on the $j \omega$ axis to get the frequency response, we obtain for the magnitude of $G(j w)$,

$$
|G(j \omega)|=\frac{4 \sin ^{2}(0.5 T \omega)}{T^{2} \omega^{2}}|C(z=\operatorname{Exp}[j \omega T])| .
$$

The phase of the frequency response is

$$
\angle G(j \omega)=-\tau \omega-T \omega+\angle C(z=\operatorname{Exp}[j \omega T]) .
$$

The magnitude and phase of error response of the entire system can be expressed in terms of just $|G(j \omega)|$ and $\angle G(j \omega)$.

Consider now a simple one-pole control system. This system at each step scales the current phase estimate by the constant $k$ (usually $0.2-0.5$ ) and adds it to the control command at the previous time step, scaled by weight $a$ (usually very close to 1.0 ). This produces the Z-transform

$$
C(z)=\frac{k}{1-a z^{-1}} .
$$

Combined with the above results, this produces an error response magnitude of

$$
|E(j \omega)|=\left[1+|G(j \omega)|^{2}+2|G(j \omega)| \cos \angle G(j \omega)\right]^{-1 / 2},
$$

where the magnitude of $G$ is now

$$
|G(j \omega)|=\frac{4 \sin ^{2}(0.5 T \omega)}{T^{2} \omega^{2}} \frac{k}{\left(1+a^{2}-2 a \cos \omega T\right)^{1 / 2}},
$$

and the phase is

$$
\angle G(j \omega)=-\tau \omega-T \omega-\tan ^{-1}\left(\frac{a \sin \omega T}{1-a \cos \omega T}\right) .
$$

These functions can be evaluated numerically. The magnitude of the error response of the AO system for $200 \mathrm{~Hz}$ 1-pole control is shown in Fig. 2, left panel. This plot shows how the various temporal frequencies in the time-varying phase aberration $\phi(t)$ get through to the residual error. The system suppresses (or is capable of controlling) temporal frequencies where the magnitude of the transfer function is less than $0 \mathrm{~dB}$. This defines the bandwidth of the system. In this case the system is running at $200 \mathrm{~Hz}$ but the bandwidth is $12.2 \mathrm{~Hz}$. Past this frequency the phase is amplified. 


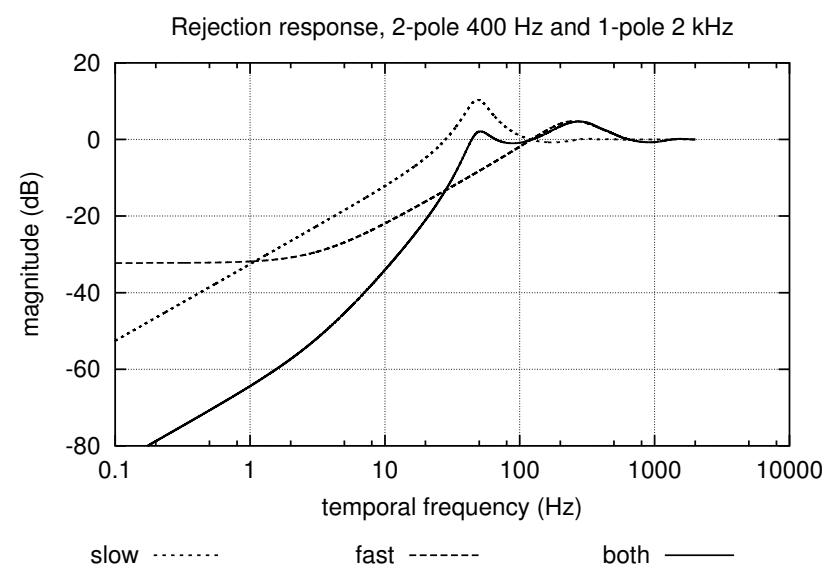

Figure 3. The interaction between the $400 \mathrm{~Hz}$ 2-pole system and the $2 \mathrm{kHz}$ 1-pole ExAO system is unfavorable. The peak in the slow system's response around $30 \mathrm{~Hz}$ is more than the ExAO system's attenuation, which drastically reduces the overall bandwidth.

\subsection{Combining two systems}

Just as one system can be modelled, the two AO systems of concern (the low-order system in front and the ExAO system) can be analyzed together. In block diagram format the two systems simply cascade. This setup is shown in Fig 1. The input to the ExAO system is just the error residual of the first system. The error response from the phase input $\phi(t)$ to the residual after the ExAO system is simply the product of the two error responses

$$
E(j \omega)=E_{1}(j \omega) E_{2}(j \omega)=\left(1+G_{1}(j \omega)\right)^{-1}\left(1+G_{2}(j \omega)\right)^{-1}
$$

The planned control rate of the ExAO system is $2 \mathrm{kHz}$ and we assume 1-pole control. Shown in Fig. 2, right panel, are the individual and combined error responses. The increase in rate simply shifts the error response farther along the frequency axis. On the $\log -\log$ plot the product $E_{1} E_{2}$ is just an addition of the curves. The ExAO system has a bandwidth of $122 \mathrm{~Hz}$ and the combined system has a bandwidth of $124 \mathrm{~Hz}$. Below the bandwidth of the slow system $(12.2 \mathrm{~Hz})$ the combined AO system corrects much better than either one individually. Above this bandwidth, the combined system still attenuates the phase error, but not as well as the ExAO system alone. This is because the slow system amplifies phase error just past its bandwidth. Above the ExAO bandwidth (122 $\mathrm{Hz}$ ) the combined system has essentially the same response as the ExAO system.

There is a tradeoff in performance between the combined system and just the ExAO system. Ignoring the stroke limitation issue, the benefit of using two systems is that low-temporal frequency errors are suppressed much better. Mid-range errors (between the slow bandwidth and the fast bandwidth) are corrected less well in the dual system. Whether this tradeoff is a good one depends upon the temporal spectrum of the phase aberration. If most of the phase power is at low temporal frequencies (which it is in the atmospheric turbulence case) the improvement in the lower band will outweigh any loss of performance in the middle band.

Care must be taken when combining the slow and fast AO systems. The above case is actually a very good scenario. A different scenario might be a slow system running at $400 \mathrm{~Hz}$ with 2-pole control instead of 1-pole. 2-pole control has a Z-transform of

$$
C(z)=\frac{k}{\left(1-z^{-1}\right)\left(1-a z^{-1}\right)}
$$

This scenario is shown in Fig. 3 In this case the 2-pole control gives more low-frequency attentuation to the slow system. However, it also leads to more amplification past the system bandwidth of $24 \mathrm{~Hz}$. This amplification is actually greater than the ExAO attenuation for some frequencies, reducing the combined system bandwidth to just $44 \mathrm{~Hz}$. Increased temporal bandwidth, which is one of the main benefits of running at a very high rate is compromized. This problem could be ameliorated by slowing down the slow system to less than $200 \mathrm{~Hz}$. It is also 
worth analyzing whether or not the peak of the slow system corresponds to sources of error, such as vibratory modes of the telescope, which might occur in the 10-40 Hz range, so as not to amplify them.

This analysis also provides insight into the question of modal removal. Should the ExAO system correct only high-order modes and leave the low-order modes to the slow system? Assuming the the variable $\phi(t)$ represents the coefficient to a mode of the phase aberration, the analysis of this section shows that two systems together will usually correct that mode better than just the slow system. This is because the added system runs at a faster rate. The converse situation is also not necessary, i.e. removing modes from the slow system. These low-order modes will require a lot of stroke, and may not be fully correctible by just the ExAO system. Secondly, unless these modes have temporal power spectra such that the power is concentrated between the low bandwidth and the high bandwidth, the combined system will be better than just the fast ExAO. Modal removal from either system is not recommended by this analysis.

In conclusion, running a fast ExAO system after a slower, low-order AO system is viable. Correction in the low frequencies will be much better than a single system alone. Care must be taken to analyze the temporal response of the low-order system and to ensure that there is adequate separation between the two control rates.

\section{THE SPATIALLY-FILTERED WAVE-FRONT SENSOR}

The in-depth analysis of the spatially-filtered wave-front sensor ${ }^{5}$ (SFWFS) will not be repeated here. Instead, the key results and properties for ExAO wave-front control will be summarized.

In the general case, AO systems operate using sampled measurements of the phase, or derivative of the phase. ${ }^{1}$ For ExAO we anticipate using the Shack-Hartmann sensor, which samples the phase gradient every subaperture width $d$ in the pupil plane. The reconstructed phase aberration is then applied as a correction, with use of wave-front correction devices that have discrete actuators. Because the phase is not bandlimited in the general case, aliasing will occur. Aliasing is a phenomenon associated with sampling that causes high-frequency signals to be measured as low frequency signals. If the wave-front is sampled spatially with period $d$ in both $\mathrm{x}-$ and $y$-directions in the pupil plane, the phase must have no spatial frequency content above $1 / 2 d$ for exact sampling and reconstruction to occur. This condition will in the general case not be met.

Aliasing will degrade the performance of an ExAO system. In particular, it is detrimental in the astronomical high-contrast imaging case, i.e. the search for faint objects close to bright stars. As discussed elsewhere, ${ }^{5,6}$ at high Strehl ratios when diffraction has been controlled, the point-spread function (PSF) outside the core approaches the power spectral density (PSD) of the phase, though not exactly. With perfect knowledge of the phase, a deformable mirror should be able to sharply attenuate the components of the phase that correspond to spatial frequencies up to $1 / 2 d$, removing scattered light at radii less than $\lambda / 2 d$ and producing a PSF with a square dark region. Removing the aliased power can result in vastly greater sensitivity to companions.

The spatial filter is implemented as a hard-edged field stop in a focal plane before the WFS. The stop is $\lambda / d$ wide (arcsec). Light scattered by spatial-frequency components above $1 / 2 d$ frequency will be beyond this field stop. It will be rejected, leading to suppression of up to $10^{6}$ in phase power. Low-spatial frequency phase errors will, however, not be contained entirely within the field stop, leading to bleeding of phase power. The better the corrected phase residual is at low-spatial-frequencies, the better the performance of the SFWFS at high-spatial frequency rejection. This makes the SFWFS ideal for the ExAO case where very high Strehl ratios will be achieved.

Performance of the SFWFS was analyzed with our end-to-end ExAO system using Fourier optics. We used static errors with no noise in closed-loop operation to isolate SFWFS performance. Two aberrations were chosen: $20 \mathrm{~cm} r_{0}$ atmospheric turbulence and the phase errors due to a segmented primary mirror (see the reference ${ }^{5}$ for details of the model.) The base-line design is based on a proposed high-order AO system for direct imaging of extra-solar planets. ${ }^{7}$ In this case we chose the upper end of the number of subapertures, with $D / d=62$ across a 10-m mirror. The nominal WFS is a Shack-Hartmann wave-front sensor with $4 \times 4$ pixels per subaperture, which was simulated with Fourier optics and with centroiding for slope estimation. The WFS uses $800 \mathrm{~nm}$ light. The phase estimation was done with the Fourier transform reconstructor. ${ }^{8}$ After loop convergence the residual error was analyzed via PSD estimation. PSFs were generated at 1.6 microns with use of a Blackman apodization 

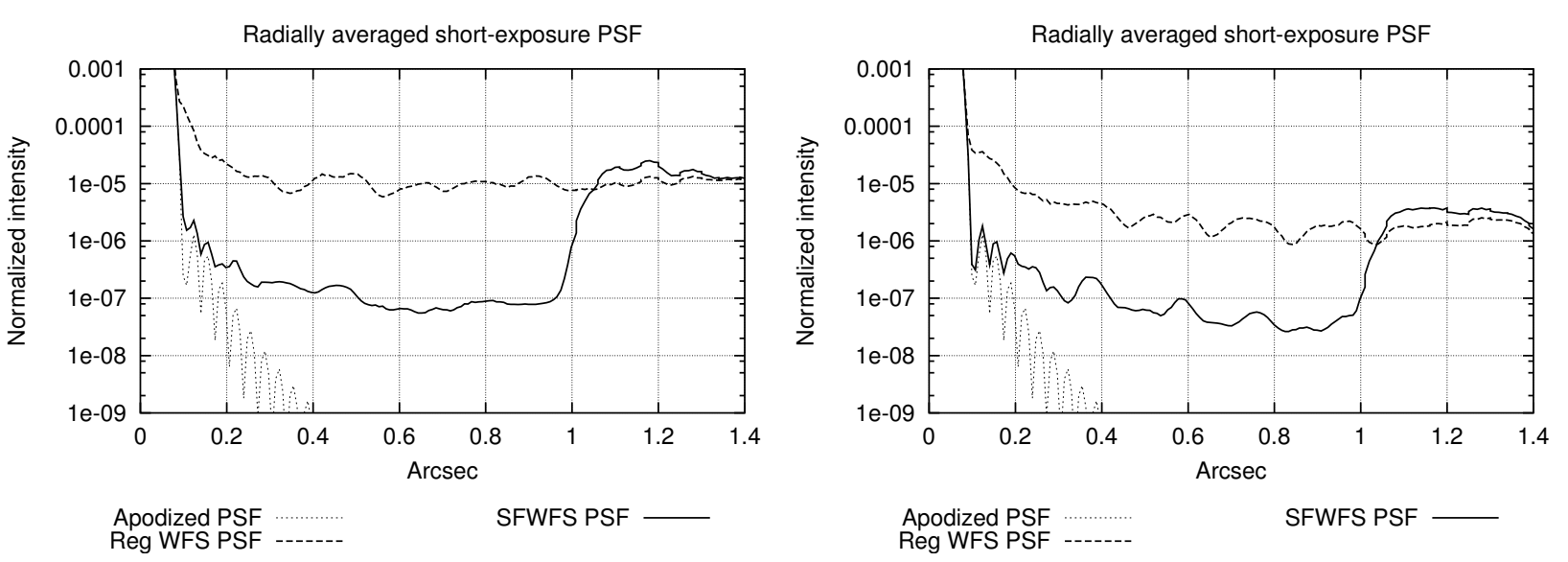

Figure 4. Apodized PSFs with and without the SFWFS in closed-loop operation on [left panel] $20 \mathrm{~cm} r_{0}$ atmospheric turbulence and [right panel] segmented primary mirror errors. Use of the SFWFS gives a 50 to 100 times reduction in PSF intensity within a large region, getting to 7 orders of magnitude less than the intensity of the PSF peak.

of the pupil. These PSFs (see Fig. 4) show that the SFWFS can lower PSF intensity by 50 to 100 times across a broad range from the central core of the PSF. This is the dark area mentioned above.

A second major benefit of the SFWFS is that is can prevent phase errors at high spatial frequencies that are introduced by the DM itself. As the following section explores, non-ideal DMs introduce power beyond the spatial frequency cutoff $1 / 2 d$. If not adequately suppressed, aliasing of this extra phase power can inhibit stable performance in closed loop with segmented DMs.

\section{DEFORMABLE MIRROR MODELS AND EFFECTS ON PERFORMANCE}

The ability of the AO system to correct the phase aberration depends in part on the response of the DM. The DM can be a monolithic continuous glass facesheet mirror, or a continuous- or segmented-surface MEMS device. To study the behavior of various DMs, we used the linear superposition model for the actuator response. In this model the response of the DM is a linear superposition of the influence functions of each actuator, which are assumed to be spatially invariant with actuator location. This makes the influence function the impulse response of the DM and the Fourier transform of the influence function its frequency response, which is a non-ideal lowpass filter. In the case of continuous facesheet DMs, this is a reasonable, but not highly accurate, model. ${ }^{9}$ For segmented MEMS devices this is a much better model, because the segments are physically decoupled.

Theoretical predictions of DM performance were based on the frequency responses of the DMs. Given an actuator spacing of $d$ on the DM, the ideal frequency response is a perfect low-pass filter with cutoff frequency of $1 / 2 d$. This would be a sinc-interpolating DM, which does not exist in practice. All other DMs are non-ideal, and we examine how their performance differs from the ideal. There are two significant manifestations of using a non-ideal DM. First, the DM can attenuate spatial-frequencies in its correctable pass-band. This leads to a lower gain in the system for those frequencies. In open loop, this means that correction of those frequencies will be only partial. In closed loop this is ameliorated somewhat, though the gain will increase the steady-state error of the system in correcting that spatial frequency.

Second, a non-ideal DM introduces high-spatial frequency errors which alias into the sensed phase when that phase is sampled. This phenomenon is more troubling than attenuation, particularly in the segmented DM case. Aliasing of the phase can increase residual error in the passband and can even lead to instability in the system. Aliasing can be mitigated with use of a spatial filter, which was described above.

Here we analyze the performance of several DM models. They are divided into two types: continuous and segmented DMs. The Fried-geomtery is used, where the average phase gradient is measured in each subaperture in a square lenslet array. The phase is then reconstructed and values are determined for the phase points at 
the corners of the subapertures. For the continuous DMs, these phase points correspond to physical actuator locations. For the segmented DMs, the phase points may or may not correspond to the actual location of the DM actuators for the segments. For reference throughout we will compare performance to the ideal low-pass filter. Its impulse response and transfer function are

$$
\begin{gathered}
h_{I}(x, y)=\frac{\sin (\pi x / d)}{(\pi x / d)} \frac{\sin (\pi y / d)}{(\pi y / d)}, \\
H_{I}\left(f_{x}, f_{y}\right)= \begin{cases}d^{2} & \left|f_{x}\right|<(2 d)^{-1},\left|f_{y}\right|<(2 d)^{-1} \\
0 & \text { else }\end{cases}
\end{gathered}
$$

\subsection{Continuous surface DMs}

For continuous DMs, we used the following influence functions. The first is the difference of two bivariate, uncorrelated gaussians. This function is a reasonable approximation, but has the flaw of being radially symmetric on a square grid of actuators. For the difference of two Gaussians, the general formulas are

$$
\begin{gathered}
h_{G}(x, y)=\left(\operatorname{Exp}\left[-a\left(x^{2}+y^{2}\right)\right]-k \times \operatorname{Exp}\left[-b\left(x^{2}+y^{2}\right)\right]\right)(1-k)^{-1}, \\
H_{G}\left(f_{x}, f_{y}\right)=\left(\frac{\pi}{a} \operatorname{Exp}\left[\frac{-\pi^{2}\left(f_{x}^{2}+f_{y}^{2}\right)}{a}\right]-k \frac{\pi}{b} \operatorname{Exp}\left[\frac{-\pi^{2}\left(f_{x}^{2}+f_{y}^{2}\right)}{b}\right]\right)(1-k)^{-1} .
\end{gathered}
$$

In our case with subaperture spacing $d$, the constants in the above equations were chosen to be $a=2.51952 / d^{2}$, $b=0.65^{2} a$ and $k=0.241801$. These constants set the influence function to be 0 at the closest neighboring actuators and capture the undershoot curvature of the surface between the first and second neighbors.

The second influence function is the ideal sinc multiplied by a gaussian. For the sinc-gaussian model, the influence function and response are

$$
\begin{gathered}
h_{S}(x, y)=\operatorname{Exp}\left[-c\left(x^{2}+y^{2}\right)\right] \frac{\sin (\pi x / d)}{(\pi x / d)} \frac{\sin (\pi y / d)}{(\pi y / d)} \\
H_{S}\left(f_{x}, f_{y}\right)=\left\{\frac{\pi}{c} \operatorname{Exp}\left[\frac{-\pi^{2}\left(f_{x}^{2}+f_{y}^{2}\right)}{c}\right]\right\} * \begin{cases}d^{2} & \left|f_{x}\right|<(2 d)^{-1},\left|f_{y}\right|<(2 d)^{-1} \\
0 & \text { else }\end{cases}
\end{gathered}
$$

In this case $c=1 / 4 d^{2}$. The convolution integral can be evaluated numerically with the error function $\mathcal{E}(x)$, which is the cumulative density function of the unit-normal random variable, such that

$$
H_{S}\left(f_{x}, f_{y}\right)=d^{2}\left[\mathcal{E}\left(\frac{f_{x}+(2 d)^{-1}}{\sqrt{c /\left(2 \pi^{2}\right)}}\right)-\mathcal{E}\left(\frac{f_{x}-(2 d)^{-1}}{\sqrt{c /\left(2 \pi^{2}\right)}}\right)\right]\left[\mathcal{E}\left(\frac{f_{y}+(2 d)^{-1}}{\sqrt{c /\left(2 \pi^{2}\right)}}\right)-\mathcal{E}\left(\frac{f_{y}-(2 d)^{-1}}{\sqrt{c /\left(2 \pi^{2}\right)}}\right)\right] .
$$

The two preceding influence functions are attempts to approximate the empircally determined response of an actuator push on a continuous DM.

Cross-sections of the influence functions for the ideal case and the continuous DMs are shown in Fig. 5, left panel. While the ideal sinc response extends out infinitely, the $h_{G}$ and $h_{S}$ approximations are limited in practical extent to the third actuator away. The small differences in shape between $h_{G}$ and $h_{S}$ correspond to large difference in frequency response. As shown in Fig. 5, right panel, the difference of gaussians model significantly attenuates low spatial frequencies and introduces more high-spatial frequency content than the sinc-gaussian model. 

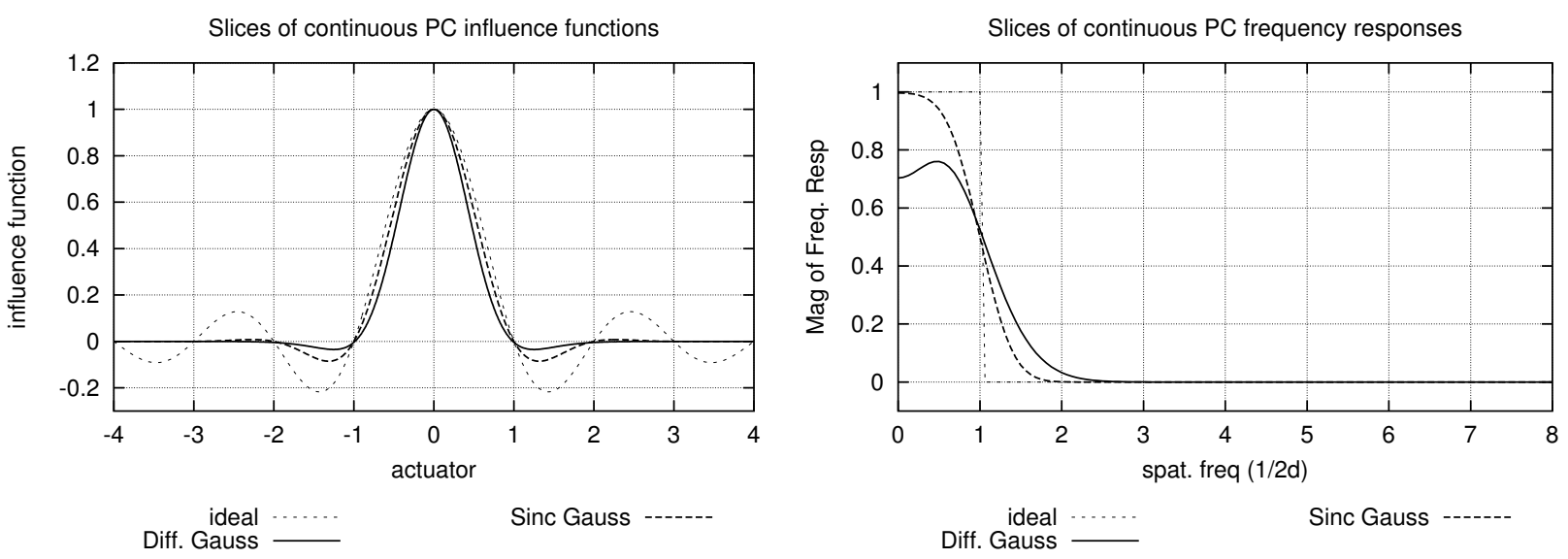

Figure 5. Continuous face-sheet DMs. [Left]: Slices through the impulse response along one axis. [Right]: Slice through magnitude of frequency response along one axis. The difference of gaussians model attenuates low-spatial frequency content and passes more high-frequency content.

\subsection{Discontinuous DMs}

For the discontinuous DMs we examine three more influence functions. Two are variations of the square-segment piston-only DM, in which a square segment the size of a subaperture moves up and down independent of neighboring segments. This is equivalent to a zero-order hold. This configuration is analyzed for the segments being centered over the phase points, and the segments begin centered in each sensing subaperture. When the segment is centered over a phase point, its value is set to that phase value. When it is centered in a subaperture, its value is the average of the four surrounding phase point values. The piston-only response, centered over the phase point, is

$$
\begin{gathered}
h_{P 1}(x, y)=\left\{\begin{array}{ll}
1 & |x|<d / 2,|y|<d / 2 \\
0 & \text { else }
\end{array},\right. \\
H_{P 1}\left(f_{x}, f_{y}\right)=\frac{\sin \pi f_{x} d}{\pi f_{x}} \frac{\sin \pi f_{y} d}{\pi f_{y}} .
\end{gathered}
$$

When the segment is shifted to the center of the subaperture and the value is the average of the four phase points, the response changes to:

$$
\begin{gathered}
h_{P 2}(x, y)=0.25\left[h_{P 1}\left(x-\frac{d}{2}, y-\frac{d}{2}\right)+h_{P 1}\left(x-\frac{1}{2}, y+\frac{d}{2}\right)+h_{P 1}\left(x+\frac{d}{2}, y-\frac{d}{2}\right)+h_{P 1}\left(x+\frac{d}{2}, y+\frac{d}{2}\right)\right] \\
H_{P 2}\left(f_{x}, f_{y}\right)=0.5\left[\cos \left(\pi d f_{x}\right)+\cos \left(\pi d f_{y}\right)\right] H_{P 1}\left(f_{x}, f_{y}\right)
\end{gathered}
$$

Note that his piston-centered model, due to the averaging of the phase points, has a response modulated every $1 / d$ in frequency.

The final segmented configuration is square tip-tilt-piston segments centered in each subaperture. In this case the segment is set to the least-squares fit of a plane to the four phase point values at the segment corners. The response and transfer function for the least-squares fit is given by a rather complex set of equations. The equation for a least-squares plane between four phase points at $(0,0, l),(d, 0, m),(0, d, p)$ and $(d, d, q)$ is $z=A x+B y+C$ for $0 \leq x \leq d$ and $0 \leq y \leq d$. The coefficients are linear combinations of the four actuators values such that $A=0.5(-l+m-p+q), B=0.5(-l-m+p+q)$ and $C=0.25(3 l+m+p-q)$. The response can then be pieced together based on shifted linear combinations of three responses

$$
h_{a}(x, y)= \begin{cases}x / d & 0 \leq x \leq d, 0 \leq y \leq d \\ 0 & \text { else }\end{cases}
$$




$$
\begin{gathered}
h_{b}(x, y)=\left\{\begin{array}{ll}
y / d & 0 \leq x \leq d, 0 \leq y \leq d \\
0 & \text { else }
\end{array},\right. \\
h_{c}(x, y)= \begin{cases}1 & 0 \leq x \leq d, 0 \leq y \leq d \\
0 & \text { else }\end{cases}
\end{gathered}
$$

such that

$$
\begin{aligned}
h_{T}\left(f_{x}, f_{y}\right)= & 0.5\left[-h_{a}(x, y)+h_{a}(x+d, y)-h_{a}(x, y+d)+h_{a}(x+d, y+d)\right]+ \\
& 0.5\left[-h_{b}(x, y)-h_{b}(x+d, y)+h_{b}(x, y+d)+h_{b}(x+d, y+d)\right]+ \\
& 0.25\left[3 h_{c}(x, y)+h_{c}(x+d, y)+h_{c}(x, y+d)-h_{c}(x+d, y+d)\right]
\end{aligned}
$$

The total transfer function is then

$$
\begin{aligned}
H_{T}\left(f_{x}, f_{y}\right)= & 0.5\left(-1+\operatorname{Exp}\left[-j 2 \pi d f_{x}\right]\right)\left(1+\operatorname{Exp}\left[-j 2 \pi d f_{y}\right]\right) H_{a}\left(f_{x}, f_{y}\right)+ \\
& 0.5\left(1+\operatorname{Exp}\left[-j 2 \pi d f_{x}\right]\right)\left(-1+\operatorname{Exp}\left[-j 2 \pi d f_{y}\right]\right) H_{b}\left(f_{x}, f_{y}\right)+ \\
& 0.25\left(3+\operatorname{Exp}\left[-j 2 \pi d f_{x}\right]+\operatorname{Exp}\left[-j 2 \pi d f_{y}\right]-\operatorname{Exp}\left[-j 2 \pi d\left(f_{x}+f_{y}\right)\right]\right) H_{c}\left(f_{x}, f_{y}\right),
\end{aligned}
$$

where the Fourier transforms of these functions are

$$
\begin{aligned}
H_{a}\left(f_{x}, f_{y}\right)= & \frac{\operatorname{Exp}\left[-j \pi d\left(f_{x}+f_{y}\right)\right]}{j 2 \pi d f_{x}} \frac{\sin \pi d f_{y}}{\pi f_{y}}\left(\frac{\sin \pi d f_{x}}{\pi f_{x}}-d \times \operatorname{Exp}\left[-j \pi d f_{x}\right]\right), \\
H_{b}\left(f_{x}, f_{y}\right)= & \frac{\operatorname{Exp}\left[-j \pi d\left(f_{x}+f_{y}\right)\right]}{j 2 \pi d f_{y}} \frac{\sin \pi d f_{x}}{\pi f_{x}}\left(\frac{\sin \pi d f_{y}}{\pi f_{y}}-d \times \operatorname{Exp}\left[-j \pi d f_{y}\right]\right), \\
& H_{c}\left(f_{x}, f_{y}\right)=\operatorname{Exp}\left[-j \pi d\left(f_{x}+f_{y}\right)\right] \frac{\sin \pi d f_{x}}{\pi f_{x}} \frac{\sin \pi d f_{y}}{\pi f_{y}} .
\end{aligned}
$$

This best-fit plane segmented model has the same transfer function as that of linear interpolation, along the $f_{x}=0$ and $f_{y}=0$ axes. When $f_{x}=0, H\left(f_{x}, f_{y}\right)$ reduces to

$$
H\left(0, f_{y}\right)=\frac{\sin ^{2} \pi f_{y} d}{\pi^{2} f_{y}^{2}} .
$$

Slices along an axis for the discontinuous DMs and the frequency responses are shown in Fig. 6. The piston-only segment model allows large amounts of high-frequency error through, which will add aliasing error to phase. The tip-tilt-piston model is much better.

\subsection{DM performance in closed loop}

These various DM configurations were tested in our end-to-end ExAO simulation code, as detailed above. The reconstructor has no knowledge of the DM and the DM model is variable.

The DM responses differ in two ways. First, some have a response closer to one in the pass-band than others. These DMs should provide better correction in closed-loop. Second, some DMs let through much more highspatial-frequency content. These will introduce extra error into the system due to aliasing and should perform much worse than other DMs. The SFWFS should improve the performance of these DMs (particularly the segmented ones.)

The same two aberrations were chosen: $20 \mathrm{~cm} r_{0}$ atmospheric turbulence and the phase errors due to a segmented primary mirror. Even in the noise-free case, the piston-only segmented DMs were unstable in closed loop. A substantial portion of this problem is due to aliasing. As shown in Fig. 6, both let through large amounts of high-spatial frequency power. A secondary problem is the fact that the Shack-Hartmann sensors are insensitive to pistons. So in the $h_{P 2}$, the sensor will measure the slope of the wave-front, but not link things up correctly in the phase reconstruction. In the $h_{P 1}$ setup, each subaperture sees a quadrant of each of four adjacent segments. If the pistons between these become significant, the spot can degenerate. 

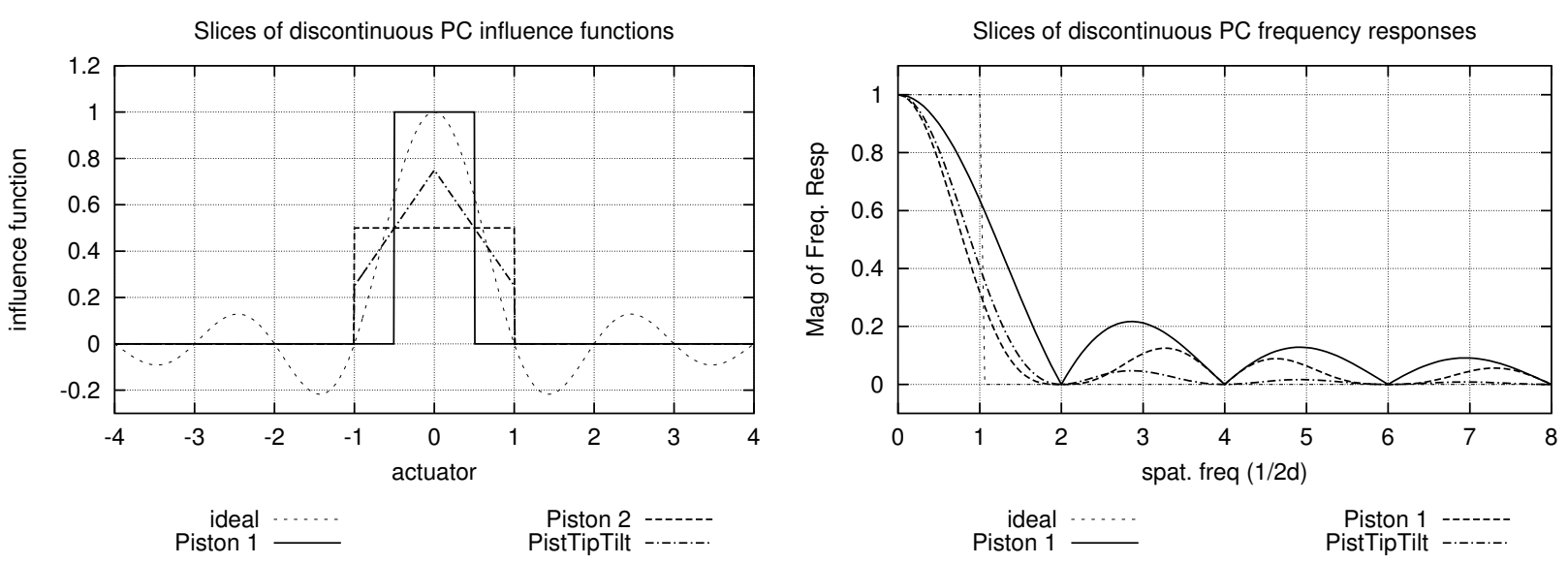

Figure 6. Segmented DMs. [Left]: Slices through the impulse response along one axis. [Right]: Slice through magnitude of frequency response along one axis. The piston-only segmented models pass large amounts of high-frequency power. The piston-tip-tilt fit is much better, with only a slight attenuation increase in the passband.
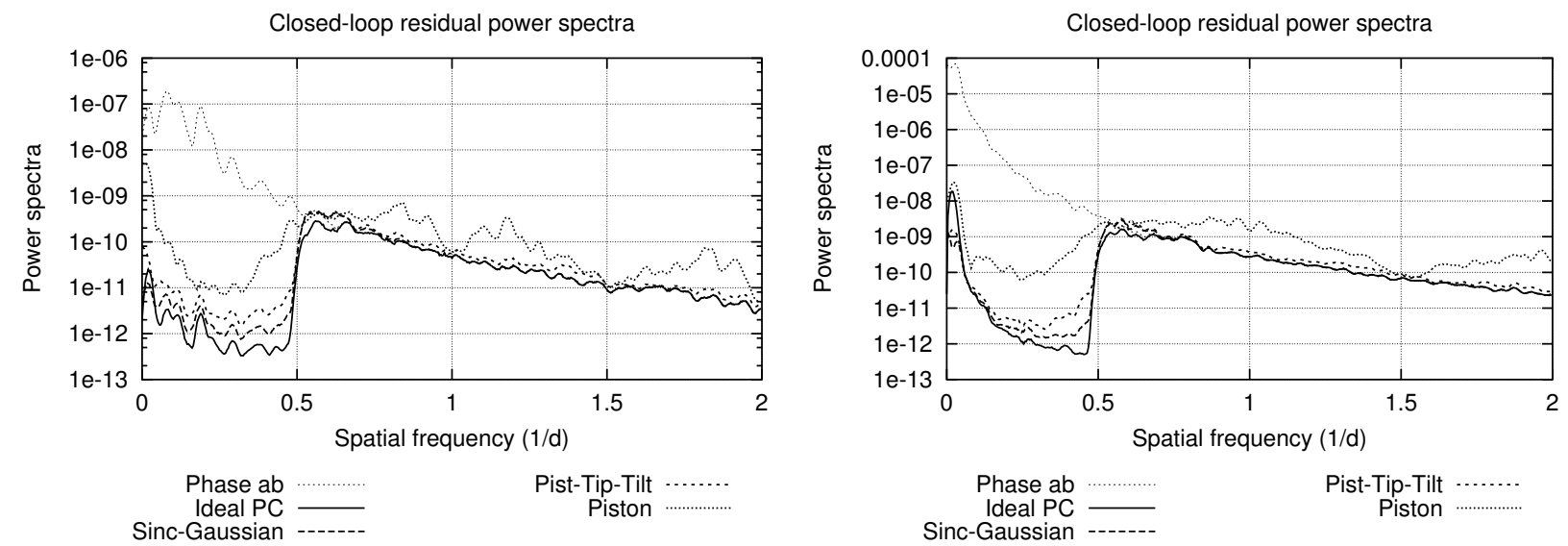

Figure 7. Radial average of residual phase PSDs for various DM models, with use of the SFWFS. [Left panel]: Segmented primary mirror errors. [Right panel]: $20 \mathrm{~cm} r_{0}$ atmospheric turbulence. In both cases, the ideal sinc interpolator is best, followed by the sinc-gaussian DM, the tip-tilt-piston segmented DM and worst of all the piston-only segmented DM.

Between the continuous DMs, the sinc-gaussian influence function performed better than the difference of gaussians. The difference between the residual PSDs and PSFs for these two are relatively small. Because neither of these influence-function models are truly accurate representations of continuous DM response, further study is indicated. Of the segmented DMs, the piston-tip-tilt model performed significantly better. Both with and without the SFWFS, it performed at close to the level of the sinc-gaussian DM. The PSDs of the residual phases (with use of the SFWFS) for the various DM models are shown in Fig. 7. In both cases the ideal DM performed the best. The sinc-gaussian shows increased power close to the cutoff of $1 / 2 d$. The piston-tip-tilt DM performs slightly worse, which is in agreement with the heuristic that a DM with a low gain in the pass-band will correct those spatial frequencies less-well in closed loop. The piston DM performs much worse in both cases. The high-spatial-frequency power that it adds in is clearly visible in both plots where the residual has more power than the input phase aberration past $1 / 2 d$. The SFWFS is a non-ideal low-pass filter on the phase. This means that aliasing will not be fully suppressed, especially given this increase in power. This leads to the extra error below $1 / 2 d$ as compared to the other DMs. For all the segmented cases, the influence-function model is more appropriate than in the continuous case. This analysis, however, has neglected diffractive effects off segment edges and gaps, which must be considered in any detailed design plan. 
In the controlled scenario of a noise-free ExAO simulation run on a static aberration, differences in DM performance are clearly visible. In these cases the piston-only segmented DMs were not stable in closed loop. Use of the SFWFS made them stable, but phase residual power is much degraded from the other DM models. The piston-tip-tilt segmented DM performed nearly as well as the best continuous DM model. More thorough study and characterization of actual devices is called for, but this result demonstrates the potential feasibility of using a segmented DM (in particular a high-order MEMS device) for ExAO phase correction.

\section{CONCLUSIONS}

We have done analysis on the technical feasibility of three key portions of ExAO wave-front control. Provided adequate characterization is done, a fast, high-order ExAO system can successfully run following a slower, loworder AO system. Spatial filtering of the wave-front phase with the SFWFS allows increased PSF sensitivity. Simple piston-only segmented DMs were shown to not be stable due to extra high-spatial frequency error, which the SFWFS can ameliorate. A piston-tip-tilt segmented DM model performed nearly as well in closed-loop operation as the standard continuous DM models on reasonable aberration profiles. These results are important steps in validating ExAO technical concepts, but must be followed up with more rigorous ExAO system design and implementation.

\section{ACKNOWLEDGMENTS}

The authors thanks J. Brase, C. Carrano and D. Gavel for their ever-useful advice. This work was performed under the auspices of the U.S. Department of Energy by the University of California, Lawrence Livermore National Laboratory under contract No. W-7405-Eng-48. The document number is UCRL JC-154631. This work has been supported by the National Science Foundation Science and Technology Center for Adaptive Optics, managed by the University of California at Santa Cruz under cooperative agreement No. AST - 9876783.

\section{REFERENCES}

1. J. Hardy, Adaptive Optics for Astronomical Telescopes, Oxford University Press, Oxford, 1998.

2. S. Olivier, "Advanced adaptive optics technology development," in Adaptive Optics Systems and Technology II, R. K. Tyson, D. Bonaccini, and M. C. Roggemann, eds., Proc. SPIE 4494, pp. 1-10, 2001.

3. N. S. Nise, Control Systems Engineering, 3rd Ed., John Wiley and Sons, New York, 2000.

4. P.-Y. Madec, "Control techniques," in Adaptive Optics in Astronomy, F. Roddier, ed., pp. 131-153, Cambridge University Press, 1999.

5. L. A. Poyneer and B. Macintosh, "Spatially-filtered wave-front sensor for high-order adaptive optics," JOSA A, p. submitted, 2003.

6. M. D. Perrin, A. Sivaramakrishnan, R. B. Makidon, B. R. Oppenheimer, and J. R. Graham, "The structure of high Strehl ratio point-spread functions," Astrophysical Journal, p. in press, 2003.

7. B. Macintosh, et al, "Extreme Adaptive Optics Planet Imager," Proc. AAS 201, p. in press, 2002.

8. L. A. Poyneer, M. Troy, B. Macintosh, and D. Gavel, "Experimental validation of Fourier transform wavefront reconstruction at Palomar observatory," Optics Letters 28, pp. 798-800, 2003.

9. S. E. Winter, J. H. Chung, and S. A. Velinsky, "Modeling and control of a deformable mirror," J. Dynamic Sys., Meas., and Control 124, pp. 297-302, 2002. 